\title{
Nasal Surgeries in Allergic Rhinitis- How far they are useful in improving Symptoms?
}

\author{
Sridhar Reddy Dandala ${ }^{1}$ Durgaprasad VN Kaza ${ }^{2}$ Narayana R Krovvidi ${ }^{3}$ \\ ${ }^{I}$ Department Of ENT, Osmania Medical College, Dr NTR University Of Health Sciences, India. \\ ${ }^{2}$ Department Of ENT, Osmania Medical College, Dr NTR University Of Health Sciences, India. \\ ${ }^{3}$ Department Of ENT, Osmania Medical College, Dr NTR University Of Health Sciences, India.
}

\begin{abstract}
Allergic rhinitis is a common condition affecting $20 \%$ of population and causes significant morbidity. It is managed conservatively. But, allergy can be associated with co-morbidities like deviated nasal septum and hypertrophy of inferior turbinate. It's sequelae include chronic sinusitis and recurrent sinonasal polyposis.. This prospective clinical study was carried out in a tertiary hospital from September 2013-2015(24 months) to see whether surgery would significantly improve their symptoms. Patients were selected $(n=50)$ with moderate to severe allergic rhinitis who were refractory to medical management alone and with significant complications. Though essential, surgery would not relieve of the patient from inherent allergy. So, standardization of indications for surgery in these conditions has been attempted. Results: Majority of patients belonged to age group11-30 with mean age of 27.84 with slightly male preponderance of 1.6:1. The daytime nasal symptom score(DTNSS) was 7.5 in pre-treatment group and was 0.24 after surgery at 3 months follow up. The ' $p$ ' value was less than 0.0001 which was statistically significant. It can be concluded that medical management alone is not sufficient in moderate to severe allergic rhinitis patients with complications and the symptoms of these patients significantly improve when combined with surgical management.
\end{abstract}

Keywords: Allergy, polyp, rhinitis, sinonasal, sinusitis.

\section{Introduction}

Allergic rhinitis is highly prevalent, allergen induced, upper airway inflammatory disease characterized by hyper active airway mucosa and episodes of symptoms, chronicity with periods of acute exacerbation. It is prevalent in up to $20 \%$ population. ${ }^{1}$ Using bothgeneric and disease-specific tools, several investigations have documented a significant impairment of quality of life in patients with allergic rhinitis . ${ }^{2}$ Patients in productive age group below 40 years are most commonly affected and a number of valuable man- hours are lost. ${ }^{3}$ Furthermore, in the past 30 years there has been a dramatic increase in prevalence of allergic rhinitis due to many contributing factors like increase in airborne pollution. Although, the etiology is uncertain, there is general agreement that it is a clinically significant disease that can lead to medical complications as nasal inflammation, nasal dysfunction can affect the upper and lower airways. Poorly controlled allergic rhinitis may lead to development of recurrent nasal polyposis (2.2\%), acute and chronic sinusitis (67\%) and bronchial asthma (15$40 \%){ }^{4}$ Poorly controlled allergic rhinitis may contribute to decrease in health related Quality of Life (HRQoL) including increased absenteeism, loss of productivity and increasing overall health care costs. Allergic rhinitis is a handicapped nose if it is associated with hypertrophied inferior turbinate, deviated nasal septum, rhinosinusitis and sinonasal polyposis.

\section{Aims and objectives}

Patients with allergic rhinitis often attend ENT out-patient department seeking relief from symptoms like nasal obstruction, chronic sinusitis and rhinitis, are found to be on medical management with anti allergicdrugs, Leukotriene inhibitors, and steroid nasal nasal sprays for a long period and found to be refractory to these treatments. These patients have mild symptoms to start with and some progress to moderate to severe form with troublesome symptoms like abnormal sleep, impairment of daily activities, sport, leisure and abnormal work/school.

A study was needed to see if nasal surgery in these medically refractory cases with moderate-severe allergic rhinitis associated with complications like sinonasal polyposis, deviated nasal septum, chronic rhino sinusitis, and inferior turbinate hypertrophy would improve the symptoms and role of surgical management in ameliorating the allergic manifestations.

\section{Methodology}

This progressive clinical study was undertaken at a tertiary hospital from September 2013- August 2015 (24 months). This prospective clinical study included patients attending ENT out-patient department and also from specialty Allergic clinic and were diagnosed as having moderate-severe form of allergic rhinitis and 
who were refractory to medical management. The symptom severity was scored on self assessment scale of 0-3 for the four cardinal symptoms of allergic rhinitis such as sneezing, itching in nose/eyes, nasal obstruction and rhinorrhoea. A total Day Time Nasal Symptom Score (DTNSS) was calculated by adding the individual scores for the four cardinal symptoms.

Inclusion criteria: A total of 50 patients $(\mathrm{n}=50)$ with moderate-severe persistent allergic rhinitis who were refractory to medical management, with co-morbidities and sequelae and patients more than 10 years,

Exclusion criteria: Patients who were less than 10 years and more than 70 years, patients who had symptomatic organic disease where surgery was contraindicated, and patients who had mild allergic symptoms.

A complete ENT examination was performed including nasal endoscopy and the findings were recorded on the basis of Lund and Mackay for polyps. An NCCT- CT scan of Paranasal sinuses was taken for each patient. The treatment protocol comprised of surgical management which was decided on the nature of complications. This included Sub mucosal diathermy for inferior turbinate hypertrophy, septoplasty for deviated nasal septum and FESS (Functional Endoscopic Sinus Surgery) for sinonasal polyposis and sinusitis. These patients were followed up monthly for 3 months and their DTNSS was recorded at the end of 3 months. Patients were advised to continue medical management for allergy.

\section{Observations And Results}

Total number of patients included in the study $\mathrm{n}=50$ from September 2013-August 2015 .

The majority of patients belonged to age group 11-30 years (60\%) followed by 31-40 years and least at 61-70 years of age. The mean age was 27.84. There was male preponderance of 1.6:1.

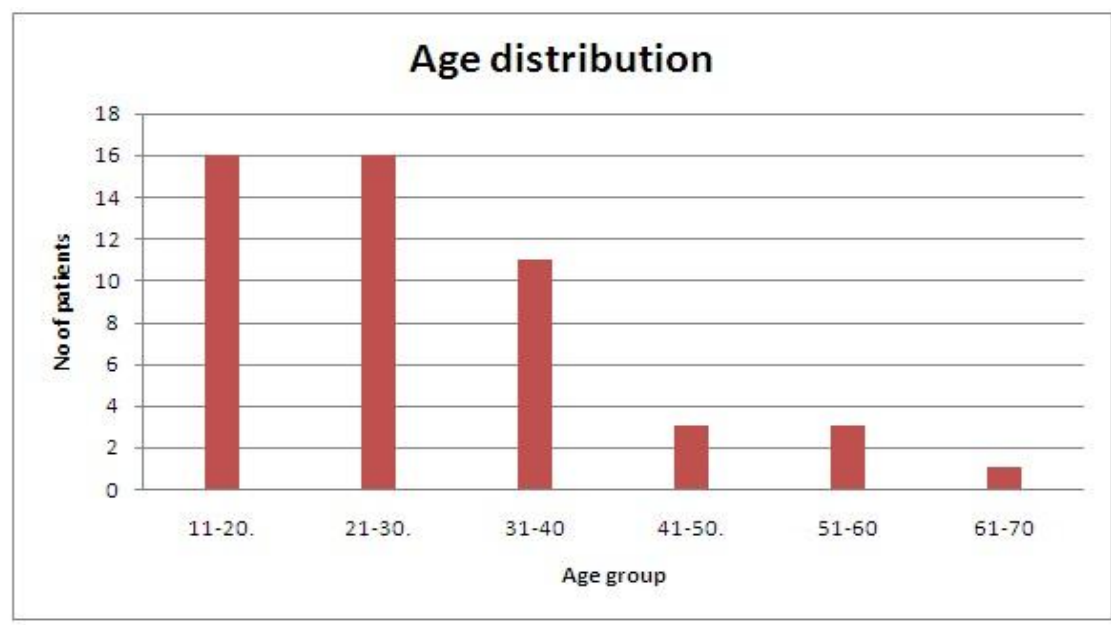

Fig.1 Age wise distribution among the patients.

Most common moderate-severe persistent symptom in the pre surgical group was sneezing followed by rhinorrhoea followed by nasal obstruction and itching of the nose.

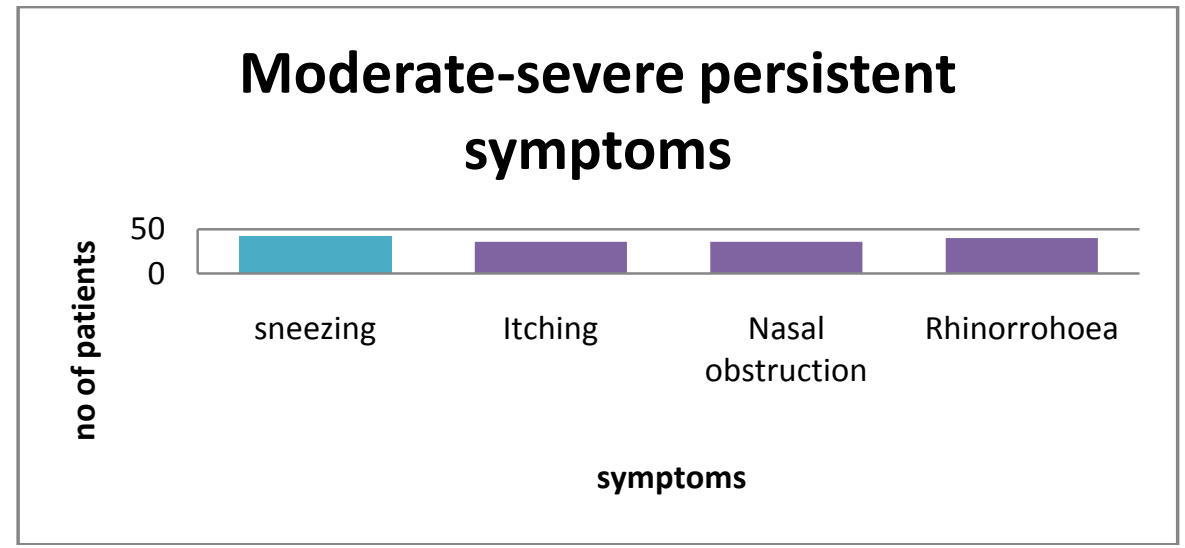

Fig.2 categorization of patients depending on the symptoms 
At the end of 3 months follow up period, mild nasal obstruction was the most common symptom observed in only 6 cases and sneezing and itching in only 3 cases.

The most common associated symptom was headache (90\%) followed by hyposmia (84\%).

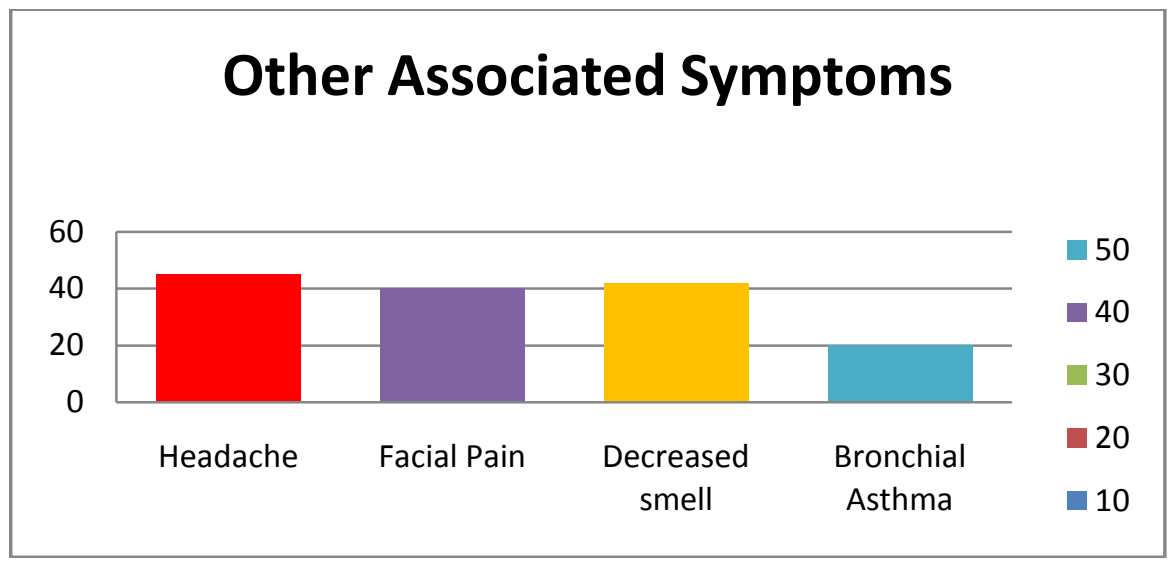

Fig.3 categorization of patients according to associated symptoms.

The most common disease noted was combination of DNS and CRS (36\%) followed by sinonasal polyposis $(26 \%)$. CRS alone was seen in $20 \%$ followed by DNS alone in $14 \%$. Inferior turbinate hypertrophy was seen in only 2 cases.

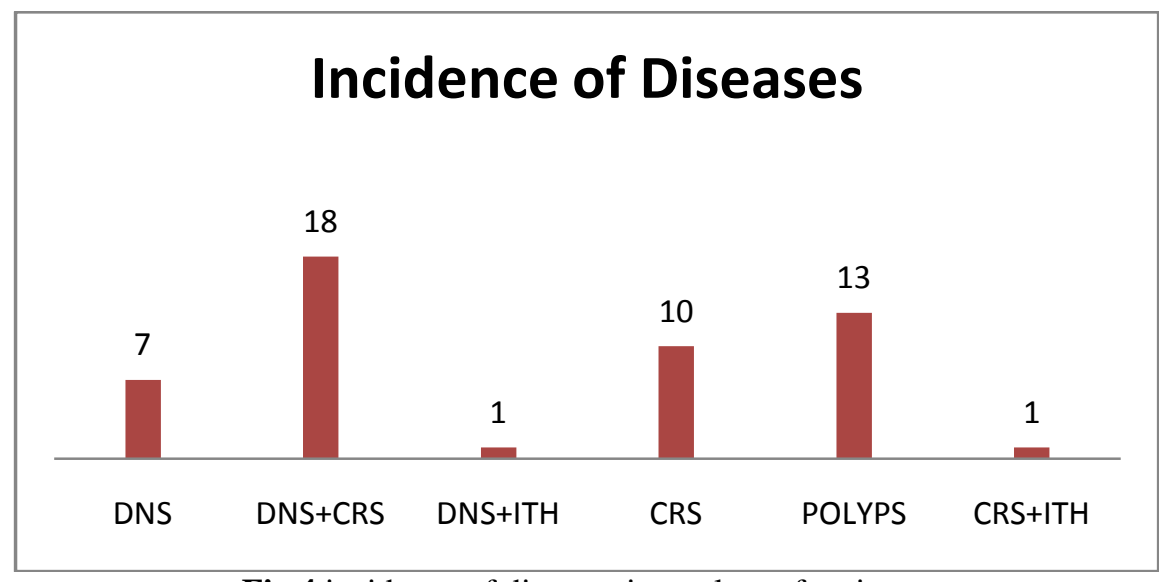

Fig.4 incidence of diseases in total no of patients.

The most common surgery performed was Functional Endoscopic Sinus Surgery alone in 50\% of patients followed byseptoplasty+Fess (32\%), septoplasty (14\%) and inferior turbinectomy (SMD) (4\%).

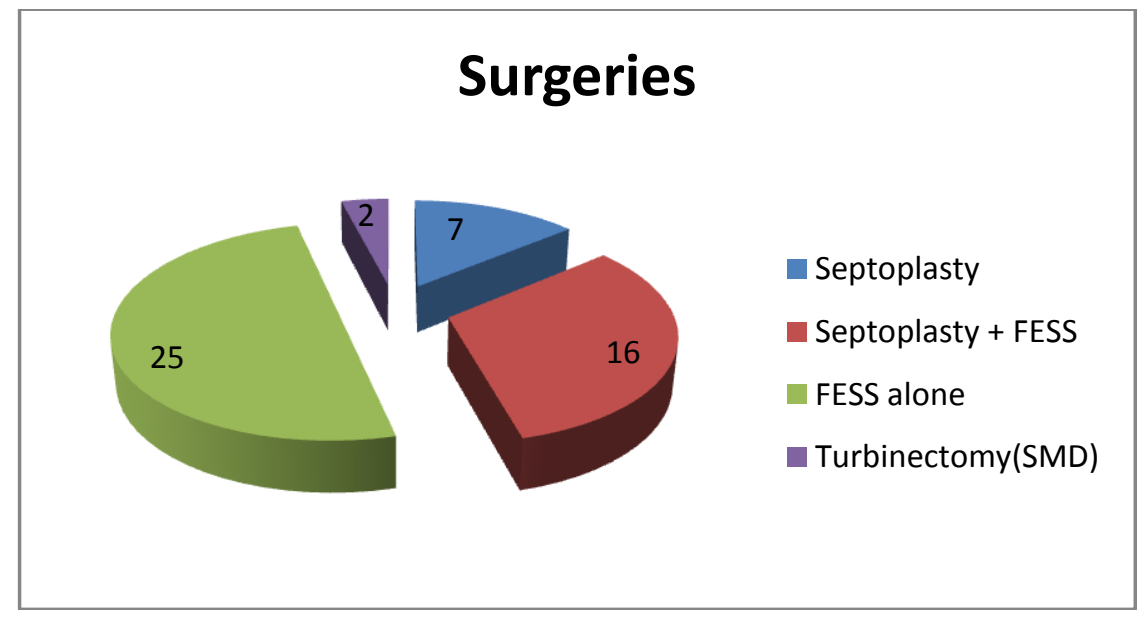

Fig.5 distribution of types of surgeries among the patients. 
The Average DTNSS in pre-treatment group was 7.5 and post- treatment after 3 months was 0.24 . The unpaired " $\mathrm{t}$ " test resulted in 'p' value was less than 0.0001 .

\section{Discussion and Conclusion}

Moderate-severe allergic rhinitis with co-morbidities was observed mostly in the relatively younger age group of 11-40 years. These patients were having mild symptoms initially and progressed to moderate to severe forms despite medical management. The reported incidence of asthma in adults with allergic rhinitis varies from $15-40 \%{ }^{4-6}$ In our study the incidence of asthma was $40 \%$ and the incidence of episodes of asthma reduced after surgery.

In patients with allergic rhinitis, conservative management including medical treatment, avoidance of allergen and immunotherapy are generally enough for controlling the symptoms, but some patients are refractory to conservative management and they need surgical treatment to improve the nasal patency and disease control to decrease allergic response. ${ }^{7}$ Nasal surgery may be needed where there is marked septal deviation or bony turbinate enlargement which makes topical nasal spray usage difficult. ${ }^{8}$ FESS may also be needed to relieve the symptoms of rhinosinusitis and consequences of intracranial and extracranial complications.

In our study, it clearly demonstrated majority of patients were relieved of nasal obstruction and the incidence of allergic episodes decreased significantly and they were mild in severity. Episodes and severity of Bronchial asthma also diminished remarkably.

The 'p' value for Pre-treatment group and post-treatment was 0.0001. By conventional criteria, this difference is considered to be statistically extremely significant. This clearly shows that patients with moderate-severe allergic rhinitiss with co-morbidities and who are refractory to conservative management alone will be benefitted with surgery and their quality of life and their allergic response will significantly improve.

\section{Recommendations}

A randomized control study with a large sample of patients needs to be undertaken with different arms of patients with mild to severe form of allergic rhinitis whether surgery is absolute necessary or relatively necessary with associated anatomical or pathological complications associated with allergic rhinitis.

\section{References}

[1]. Bousquet J, Van Cauwenberge P et al, Allergic Rhinitis and it's impact on Asthma, Journal of Allergy and Clinical Immunology, 108, 2001, S147-334.

[2]. Bousquet J, Bullinger M, Fayol C, et al: Assessment of quality of life in patients with perennial allergic rhinitis with the French version of the SF-36 Health Status Questionnaire. J Allergy Clin Immunol 94:182, 1994.

[3]. Mechanism and treatment of allergic rhinitis. IN: Durham SR, Editor, Scott \& Brown's Otolaryngology, $6^{\text {th }}$ Edvol.4, Oxford: Butterworth-Heinemann: 1997

[4]. Huse IM, Hartz SC, Russel MW, Piercey G, Weiss S. Allergic Rhinitis may worsen asthma symptoms in children; the international asthma outcome registry. Am J Respir Crit Care Med 1996;155:A860

[5]. Settipane RJ, Hagy GW, Settipane GA. Long term risk factors for developing asthma and allergic rhinitis: a 23 year follow up study of college students. Allergy Proc 1994; 15:21-25

[6]. Pariente PD, LePen C, Los F, Bousquet J. Quality of life outcomes and the use of antihistamines in a French national population based sample of patients with perennial rhinitis. Pharmacoeconomics 1997;12(5):585-595

[7]. Yoo JB, Yoon JH. Surgical Management of Allergic rhinitis. J. Korean Med Assoc. 2004 Jul; 47(7):672-678

[8]. Shahid Ali Shah, Tahira Sajid, Muhammad Asif et al. Evaluation of efficacy of management protocol in allergic rhinitis. Ayub Med Coll Abbottabad 2007; 19(3). 